\title{
A novel self-aligning mechanism to decouple force and torques for a planar exoskeleton joint
}

\author{
J. F. Schorsch ${ }^{1}$, A. Q. L. Keemink ${ }^{2}$, A. H. A. Stienen ${ }^{2}$, F. C. T. van der Helm ${ }^{1}$, and D. A. Abbink ${ }^{1}$ \\ ${ }^{1}$ Department of BioMechanical Engineering, Technical University Delft, Delft, the Netherlands \\ ${ }^{2}$ Laboratory of Biomechanical Engineering, University of Twente, Enschede, the Netherlands
}

Correspondence to: J. F. Schorsch (j.f.schorsch@tudelft.nl)

Received: 23 December 2013 - Revised: 27 May 2014 - Accepted: 18 June 2014 - Published: 6 August 2014

\begin{abstract}
The design of exoskeletons is a popular and promising area of research both for restoring lost function and rehabilitation, and for augmentation in military and industrial applications. A major practical challenge to the comfort and usability for exoskeletons is the need to avoid misalignment of the exoskeletal joint with the underlying human joint. Alignment mismatches are difficult to prevent due to large inter-user variability, and can create large stresses on the attachment system and underlying human anatomy. Previous self-aligning systems have been proposed in literature, which can compensate for muscle forces, but leave large residual forces passed directly to the skeletal system. In this paper we propose a new mechanism to reduce misalignment complications. A decoupling approach is proposed which allows large forces to be carried by the exoskeletal system while allowing both the muscle and skeletal joint force presented to the user to be compensated to any desired degree.
\end{abstract}

\section{Introduction}

Exoskeletons have been proposed to augment strength (Makinson, 1971; Zoss et al., 2006), enhance endurance (Lockheed Martin, 2012; Raytheon, 2012), and restore lost abilities (Berkeley Bionics, 2012; Jezernik et al., 2003; Stienen et al., 2007). These applications span military, (Zoss et al., 2006; Lockheed Martin, 2012; Raytheon, 2012), industrial (Makinson, 1971), and medical (Argo, 2012; Berkeley Bionics, 2012; Jezernik et al., 2003; Stienen et al., 2007) fields - each with their own challenges; but there exists a common subset of design challenges inherent to all exoskeleton systems. One of these challenges is how to fit the exoskeleton system to the operator. This problem of fit is of particular difficulty for anthropomorphic exoskeletons (Schiele and van der Helm, 2006; Stienen et al., 2009), as they are typically attached to each limb segment of the user. A misalignment between a joint in a rigid exoskeleton and the corresponding biomechanical joint in the human operator produces unexpected and potentially dangerous internal joint forces on the human as well as potential forces on the humanrobot physical interface (Schiele and van der Helm, 2006) that couple the operator to the mechanism. Furthermore it has been shown that the attachment pressure has a large ef- fect on comfort, mental load, physical demand, and effort (Schiele, 2009). There have been many different approaches to solving this issue which can be subdivided into three major classes; manual adjustment, compliant mechanisms, and kinematic redundancy. The first class of approach is simply manual linkage/patient adjustment (Evryon, 2012) - this is the most commonly used approach. In this approach, a technician will manually adjust the exoskeleton linkage lengths and restraints in an attempt to align the exoskeleton joint with the joint of the specific user. This approach can give good results, provided the degree of motion through the joint closely approximates a one degree of freedom hinge (Schiele and van der Helm, 2006; Colombo et al., 2000), and the skill of the technician is high. One of the main downsides is that this method can be time consuming, and has to be repeated each time a different user employs the exoskeleton. Additionally, choosing to use manual linkage adjustment requires careful consideration for the type of control and the placement of force and torque sensors (De Rossi et al., 2001); in that admittance can be controlled at the locations of the force sensors, while impedance control requires an accurate knowledge of the robot configuration and from that, an accurate dynamic predictive model (Kooij et al., 2006). The manual 
adjustment type systems may be considered a kinematically redundant system, in that the points of manual adjustment to represent a kinematic redundancy, but while in operation those degrees of freedom are locked, and thus should be considered separate from the true kinematically redundant systems.

The second approach commonly used, especially in rehabilitation applications (Argo, 2012; Stienen et al., 2007; Colombo et al., 2000) is not to avoid misalignment itself, but to make the restraint and attachment system compliant. This built-in compliance is assumed to be non-detrimental to performance and at least adequate for the comfort of the user. However, the comfort is not optimal due to resulting forces, and more importantly; reduced device stiffness is closely related to reduced force feedback bandwidth (Pratt et al., 1997) which may have implications for the use of high fidelity haptic force feedback, which may be necessary for the learning or rehabilitation process. For example, Wildenbeest et al. (2013) have shown that a reduction in haptic fidelity can impact both motor learning and skill generalization - both critical aspects of exoskeleton usage in an application.

A third approach is to use a kinematically redundant system such as in Lockheed Martin (2012), Argo (2012), Schiele and van der Helm (2006) and Colombo et al. (2000). In such a system degrees of freedom exist in the exoskeleton that are not present in the human operator. This allows the exoskeleton, when coupled in parallel to the human user, to be properly constrained. The kinematically redundant systems can be further subdivided into self-aligning systems and alignment free systems. A self-aligning mechanism, such used by Schiele (2009) provides excellent fitting ability; however, passive linear-motion joints do not allow for large forces to be transferred through the exoskeleton, and can introduce non-uniform inertial properties throughout the usable workspace. Conversely, in alignment free systems an additional actuator and sensors capable of controlling the DOF are required, with the associated mass, power, and control penalties inherent to such a solution. Further, such a system typically does not share a per-segment kinematic relationship with the limb segments of the operator, making multi-point contact with the operator contraindicated.

This creates a design constraint, where redundant DOF systems that need to provide high levels of force through the exoskeleton must make use of powerful actuators at each and every exoskeleton joint, and can require significant engineering design to maintain stability across the entire range of movements and joint geometries.

We propose a self-aligning joint structure that may allow for the high force transfer potential of completely powered systems with the lower actuator count and simpler control design of passive DOF systems.

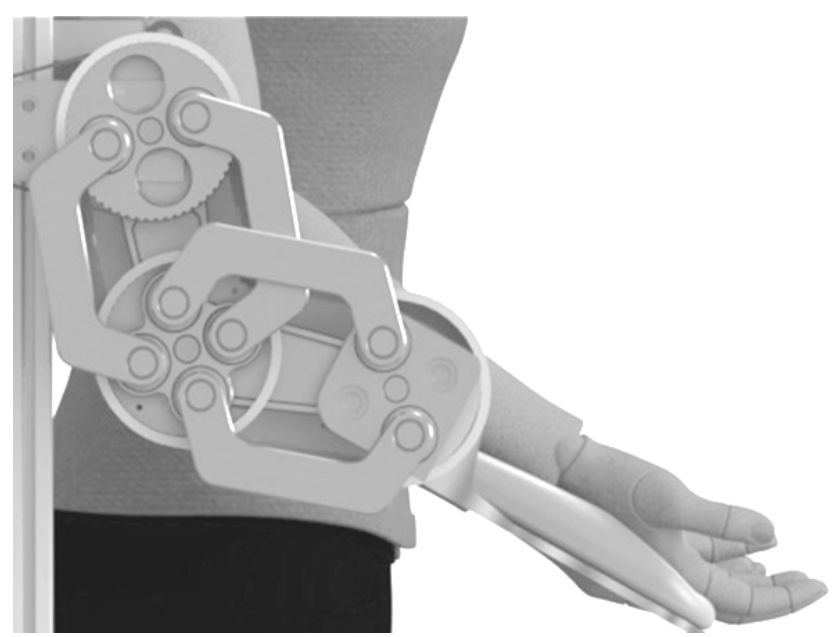

Figure 1. CAD concept of gravity compensation and torque assistance mechanism used as an elbow support exoskeleton.

\section{Design and analysis}

\subsection{Design}

In this paper, we discuss a type of planar mechanical linkage, for use in exoskeleton devices (Fig. 1), which allows both forces and torques to be passed across a virtual center of rotation, which is not known a priori. This center of rotation can exist arbitrarily at a point described within the working area of the linkage. We will show that by providing independent torque and force compensation components it is possible to both provide augmented force output to the desired load and to maintain physiologically appropriate loading of the underlying joint. In contrast to a purely passive alignment scheme, this mechanism allows for transfer of large forces and high working loads due to the novel configuration of the mechanism.

An additional advantage of the mechanism described in this paper is that forces and torques are completely decoupled from the local mechanical configuration of the mechanism. This eliminates the need for position and force sensing within the mechanism, and furthermore, does not require knowledge of the location of operator's joint, relative to the mechanism, as long as the operator's joint lies within the working range of the mechanism.

Forces and torques applied to the end effector of the linkage can also be partially decoupled. Forces which are applied through this mechanism create an apparent moment about the supported physiological joint, which need to be taken into account, while moments applied do not create additional force requirements. The degree to which the applied force is decoupled from applied torque is dependant entirely on the ratio of the amount of misalignment accommodated by the mechanism to the length of the proximal limb supported. This torque and force decoupling characteristic allows the device to be tailored for specific loading conditions, 


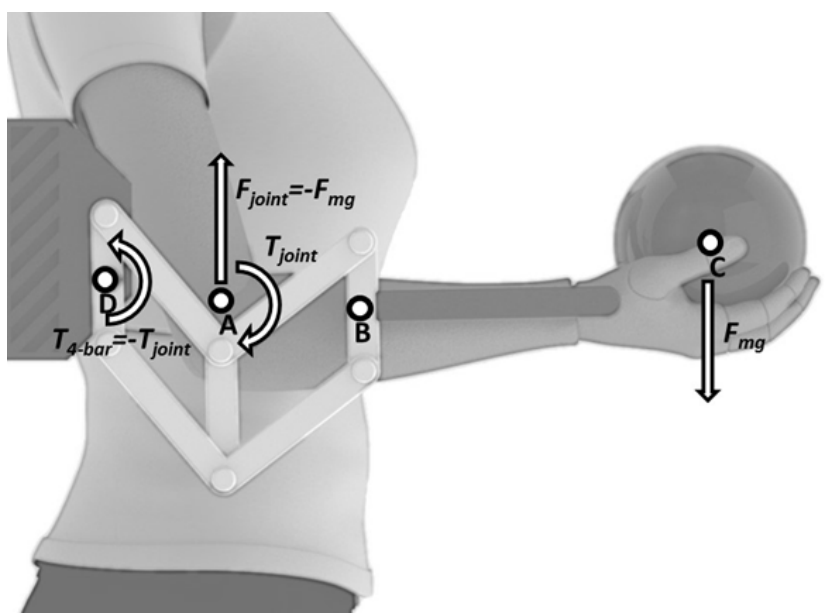

Figure 2. Example of serial 4-bar mechanism used for an elbow support exoskeleton. The user's elbow join is located at A, a load is applied at point $\mathrm{C}$, and point $\mathrm{B}$ is located arbitrarily along the line AC. The load at $\mathrm{C}$ creates a torque, $T_{\text {joint }}$ about point $\mathrm{A}$. The 4-bar mechanism is anchored at point D. $F_{\text {joint }}$ is the resultant force needed to support the load.

such as pure weight compensation, combined weight and inertia compensation, or targeted joint loading scenarios, independent of the basic mechanical design. This allows the same mechanical design to be generally applied for strength enhancement, endurance improvement, or for rehabilitative purposes.

\subsection{Predecessors}

The mechanism as discussed in this paper can be considered a novel combination of two existing, well understood, types of mechanisms:

1. a double four-bar parallelogram mechanism, such as used in the LIMPACT rehabilitation system (Stienen et al., 2009),

2. an energy-free gravity compensation mechanism as described by Tuijthof and Herder (2000).

\subsubsection{Predicate 1}

This type of mechanism can transfer torques from the ground or actuator through the linkage to a proximal end effector. If not attached to a user, the linkage is under-constrained. When the mechanism end effector is attached to a user through a restraint, the assembly becomes properly constrained.

For evaluating the loading of the human elbow during static equilibrium, the net forces and moments on the system must both sum to zero, and it can be seen that in the case (Eq. 1a) of the serial arrangement of a pair of four-bar parallel mechanisms, (see Fig. 2) the mechanism is capable

\section{B}

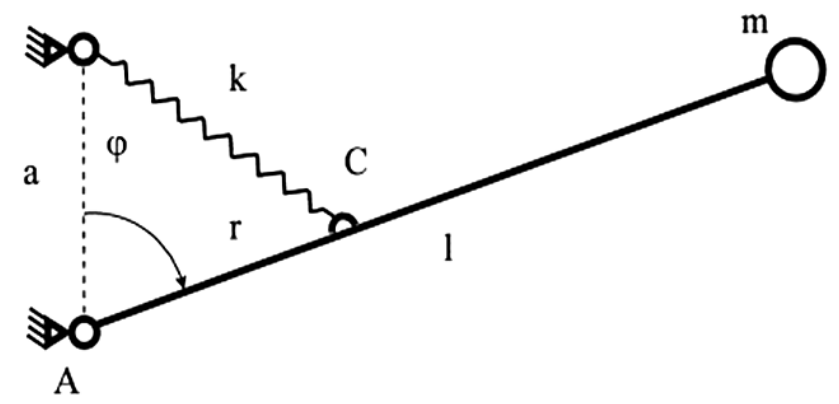

Figure 3. akr type spring gravity compensation mechanism, as introduced in Tuijthof and Herder (2000), reprinted with permission. Mass $m$, supported by bar $l$, acted on by gravity, $g$, can be exactly balanced at any point in its rotation about point A by a zero free length spring with stiffness $k$ attached to ground reference at a vertical distance $a$, and at point $r$ along bar $l$. For practical implementations spring $k$ either must act through some type of cable structure, to allow the approximation of zero free length, or some other mechanical construct must be used to allow for zero free length approximation. The design of such structure can be non-trivial, especially in constrained workspace.

of reducing the torque created by the muscles about the elbow to zero. There is a zero sum relationship between the torque borne by the mechanism and the torque at the human joint, such that the torques created by the applied load can be distributed as desired to the human joint by increasing or reducing the amount of torque provided by the exoskeleton.

$T_{4-\text { bar }}=-m_{\text {object }} \times g \times l_{\overline{\mathrm{AC}}}$

$F_{\text {joint }}=-m_{\text {object }} \times g$

By examining the balancing of forces (Eq. 1b) it is shown that a double four-bar mechanism which reduces the residual moment about the elbow to zero will leave a residual force on the elbow that is antiparallel and proportional to the weight of the load. This is contrary to the expected loading pattern experienced during unaugmented lifting, and could result in injury or unexpected control activity by the human operator.

During unaugmented lifting, the force created on the humeroulnar joint is compressive and of significantly larger magnitude than the weight of the object being lifted (Stormont et al., 1985). When changing both the direction and magnitude of the force experienced at the elbow there are two potentially important physiological effects. The first effect is the potential to increase the rates of musculoskeletal injuries. Kumar clearly makes the link that musculoskeletal injuries occur at a higher rate when the natural mechanical order of a task is disrupted (Kumar, 2001). It is unknown to the extent that disrupting the expected loading pattern by use of a double four-bar mechanism would result in increased injuries. The second effect experienced is in creating a scaled force in the opposite direct to what is expected during normal movement, and will require a new muscle activation pattern 


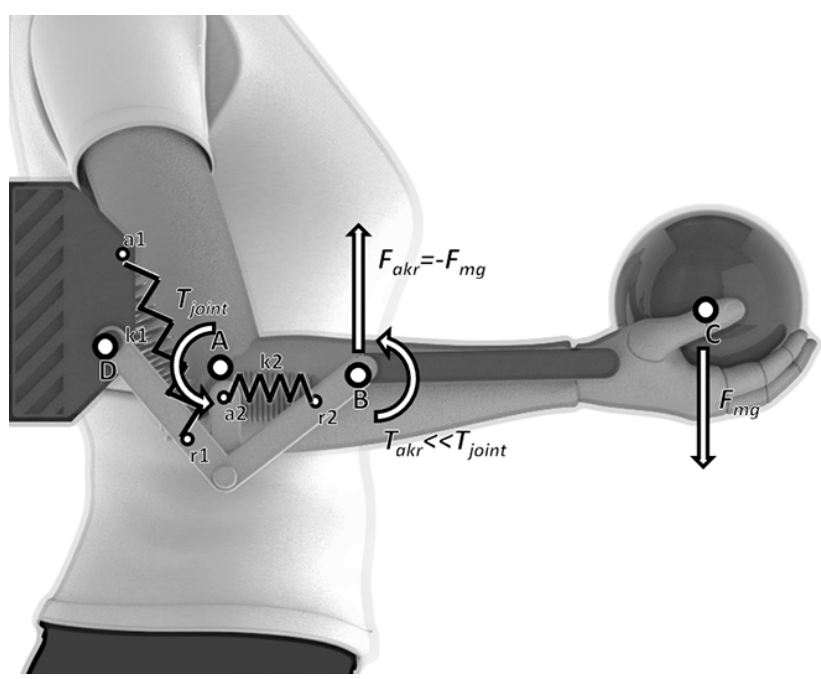

Figure 4. Example of serial akr mechanism used for an elbow support exoskeleton. The user's elbow join is located at A, a load is applied at point $\mathrm{C}$, and point $\mathrm{B}$ is located arbitrarily along the line AC.

in musculoskeletal joints more proximally located along the kinematic chain, in order to produce a specific desired movement.

In addition to the physiological complications, there are mechanical and control considerations to take into account. The moment supplied by the actuator through the serial fourbar mechanism is purely dependant on the location and magnitude of the load, relative to the elbow joint of the user, not the current mechanical configuration of the parallel mechanism. This makes it difficult to estimate the actual mass of the load based on torques or forces perceived at the mechanism, and requires direct sensing of the load, or precise knowledge of the user's anatomy, which may be difficult to obtain.

\subsubsection{Predicate 2}

The second predecessor mechanism is the so-called "akr" type of energy free gravity compensation mechanism as described by Tuijthof and Herder (2000) (Fig. 3). This type of mechanism relies on ideal linear spring behaviour to precisely compensate a fixed mass at a given distance. As the mass $m$ translates along the arc described by $l$, it stretches the spring $k$ attached at point $r$ in such a way to allow the system to remain energy neutral; e.g., the system provides a force perpendicular to $l$ and through $m$ with the component aligned parallel to $\mathrm{AB}$ remaining constant. It is possible to connect multiple single-link akr type mechanisms in series (see Fig. 4) to give an improved range of motion, and eliminate any force vector components that are not parallel to AB. In this type of serial connection, the endpoint of the proximal akr mechanism acts as the reference grounding point for the more distal akr mechanism. An important consideration in the design of serial akr mechanisms is the cancela-

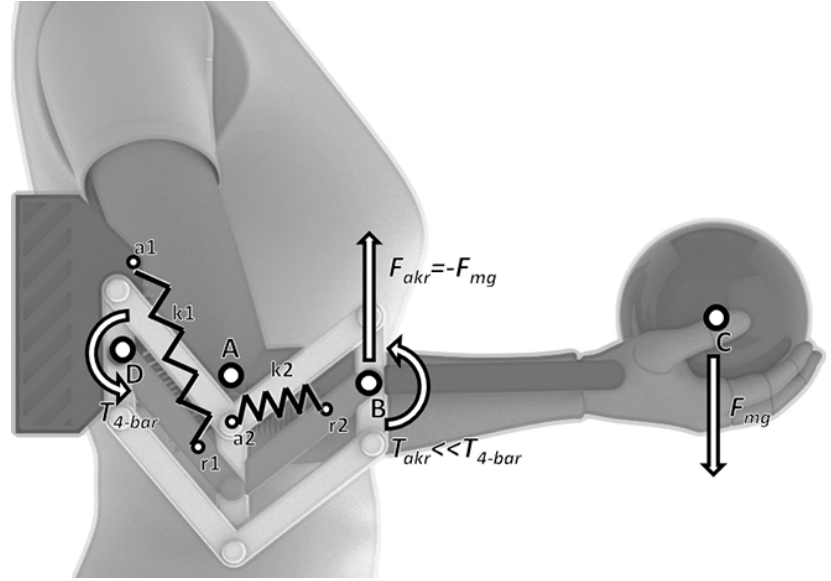

Figure 5. Example of combined mechanism used as an elbow support exoskeleton. The user's elbow join is located at A, a load is applied at point $\mathrm{C}$, and point $\mathrm{B}$ is located arbitrarily along the line AC. All forces supplied by the mechanism are explicit. Compensation case illustrated is for $100 \%$ compensation. Residual forces left to the operator at point A can be controlled to appropriate level.

tion of force components perpendicular to $\mathrm{AB}$, allowing for a zero net energy cost for displacements in the entire working plane. Potential energy lost or gained via vertical displacement is stored or recovered simultaneously in the springs for both akr mechanisms, while the energy is only transferred between springs when the system is displaced horizontally. The key feature which allows for the novelty of the combined mechanism is that the theoretical working area for two akr type compensation mechanisms working in series is the same as for a serial pair of four-bar parallelogram mechanisms. The static behaviour of a realization for such system with two serial connected akr mechanisms is described by Eq. $(2 a-b)$

$$
\begin{aligned}
& T_{\text {joint }}=-m_{\text {object }} \times g \times\left(l_{\overline{\mathrm{AC}}}-l_{\overline{\mathrm{AB}}}\right) \\
& F_{\text {joint }} \equiv 0
\end{aligned}
$$

This mechanism is capable of creating a consistently aligned force vector through the supported limb segment regardless of the attachment point of the mechanism, and also provides a degree of moment reduction, depending on the relative positions of the attachment point, relative to the physiological joint it is supporting (Eq. 2b).

\subsection{Implementation}

Each mechanism discussed previously is capable of providing one component of the solution for equilibrium; e.g. forces and moments respectively for the serial akr mechanisms and double four-bar mechanisms. The equations for equilibrium could be satisfied while controlling both the residual joint and muscle loads, if the endpoints of both mechanisms could be coupled through a joint that allowed rotational freedom, 
but no translational freedom. A simple rotational bearing provides exactly such a connection. This creates a parallel arrangement of the double four-bar mechanism and the serial akr compensation mechanism, (Fig. 5). Equations 3a-1 show the solution for the static equilibrium of such a system.

$$
\begin{aligned}
& \sum F_{\mathrm{A}}=0 \\
& F_{\text {joint }}+F_{4-\text { bar }}+F_{\text {akr }}+W_{\text {object }}=0 \\
& W_{\text {object }}=m_{\text {object }} \times g \\
& F_{4-\text { bar }} \equiv 0 \\
& F_{\text {akr }} \equiv-m_{\text {object }} \times g \\
& F_{\text {joint }}=0 \\
& \sum T_{\mathrm{A}}=0 \\
& T_{\text {joint }}+T_{4-\text { bar }}+T_{\text {akr }}+T_{\text {object }}=0 \\
& T_{\text {object }}=m_{\text {object }} \times g \times l_{\overline{\mathrm{AC}}} \\
& T_{\text {akr }}=m_{\text {object }} \times g \times l_{\overline{\mathrm{AB}}} \\
& T_{\text {joint }} \equiv 0 \\
& F_{4-\text { bar }}=m_{\text {object }} \times g \times\left(l_{\overline{\mathrm{AC}}}-l_{\overline{\mathrm{AB}}}\right)
\end{aligned}
$$

\section{Discussion}

The mechanism described in this paper has many potential applications for use in exoskeletal systems, such as transferring large forces to the ground in load bearing applications, providing reduced loading of damaged joints for rehabilitation orthotics, or for providing physiologically appropriate increased loading of bones to counter disuse atrophy. It provides a mechanism for use as a prismatic self-aligning exoskeleton joint within a described planar area. Decoupling the application of force and moments from each other creates a method to design a planar self-aligning exoskeleton joint in which both the forces in the muscles and at the joint of the user can be compensated or controlled independently. It is important to note that in the derivation of the equations of static equilibrium, the complete reduction of forces and moments at the joint was chosen for illustrative purposes. Any combination of residual joint force and torques can be designed for, depending on a particular application or experimental purpose.

There are some practical limitations to implementation of the parallel 4-bar linkage used for torque transmission. If an actual mechanical four-bar mechanism is used, when any pair of links become collinear, a mechanical singularity is reached, which leads to kinematic uncertainty. As this mechanical singularity is approached, internal forces become very high, relative to the amount of torque transmitted from the input to the output. This limits the practical working area to a smaller subset of the theoretical maximum, dependent on the initial configuration of the parallel four-bar mechanisms. By using a pseudo four-bar mechanism, such as a capstanand-cable or a belted pulley (Morita et al., 2003) anchored through rotational joints to the akr subassemblies, this mechanical singularity can be avoided.

It was noted that both the double four-bar mechanism and the serial akr mechanisms have identical working areas, as long as the effective linkage length is the same. Further, by keeping the individual linkage segments equal for both mechanisms, each point in one mechanism segment will remain in exactly the same position relative to the other. This allows further coupling of the endpoints of parallel linkage segments through rotational bearings. This coupling of the intermediate endpoints is not strictly necessary from a kinematic standpoint, however, it would allow the use of each 4-bar parallelogram as member $l$ of the akr compensation mechanism or vice versa, allowing for a reduction in the number of parts, as well as reducing the volume of the mechanism for a given compensation weight.

If the parallelogram linkages are used as a component of an akr mechanism that makes use of a pulley system (Morita et al., 2003) to transfer force to the more distal akr unit, care must be taken to maintain proper pretension of the cable or belting solution. Improper design of the relative linkages can result in overstress of the load carrying belt, which can lead to breakage, loss of load carrying ability, and premature wear.

If the 4-bar parallelograms are not used as members in the akr mechanism, and the two systems are attached together directly at the intermediate pivot point through rotational bearings, an over-constrained system is created. This can result in very high internal forces and large asymmetric loads on the coupling bearings. The magnitudes of the internal forces are purely dependent on the design of the connecting linkages and the relative precision of the rotational center distances. It is recommended that care be taken in the design of the akr mechanism to reduce the internal forces if intermediate coupling is attempted.

This mechanism was realized in hardware, according to the design shown in Fig. 6. This realization makes use of an intermediate endpoint coupling arrangement for the fourbar parallelograms and akr mechanisms. This implementation was chosen to allow use of a synchronous belt transmission to transmit force to the second of the akr linkages while still maintaining proper pretension on the synchronous belt. A compliant bushing is used around the bearing connection between the akr linkages and the double-parallelogram linkage to reduce the internal forces produced on the bearings by tolerance induced misalignment. The design decision to use a synchronous belt as part of the akr mechanism was to make the effective attachment point of both akr mechanisms coaxial. This allows the springs which drive both akr mechanisms to be physically remote from the actual joint placement, with use of a cable routing system to transmit the spring force to the akr mechanisms.

Assembly of the described mechanism is challenging. Bearings in the 4-bar mechanism were pre-compressed via heat shrink fitting of the mounting structure around the bearing race. This allows for precise control of the bearing 


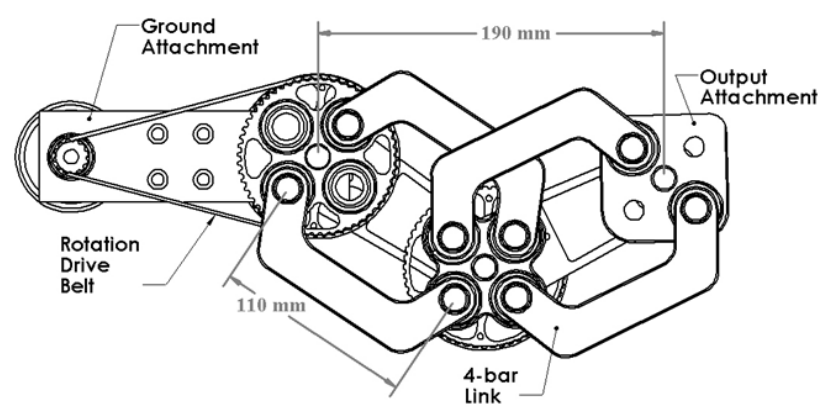

The mechanism described in this paper can be kinematically expanded through straightforward conceptual alterations. By using a parallelipiped in place of the one of the serial four-bar mechanisms, with a similar expansion for the akr type gravity compensation mechanism, it would allow accommodation of joint misalignment along all three Cartesian axis, as opposed to the purely planar mechanism described in this paper. This kinematic expansion is a non-trivial engineering challenge, and should be carefully approached.

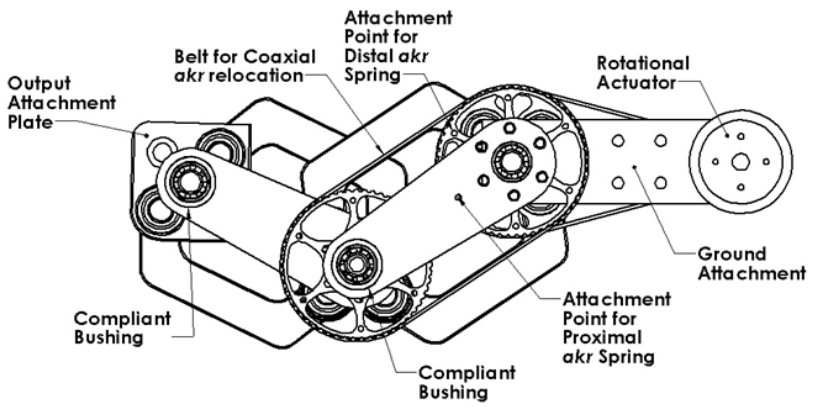

Figure 6. Technical Illustration for the mechanism as constructed. Illustrated from both sides. Top illustrates primarily the 4-bar component of the mechanism, while bottom illustrates the akr mechanism. This iteration will accommodate up to $10 \mathrm{~cm}$ of misalignment, without restriction of motion. Mechanism as designed to compensate up to $100 \mathrm{~kg}$ of applied load. Many structural members are significantly oversize to simplify manufacture and construction. All rotational joints for 4-bar linkages are supported by cylindrical roller bearings (SKF Bearing) rated to $13 \mathrm{kN}$ dynamic loading. Rotational joints for akr mechanism are supported by standard ball bearings (SKF Bearing). Teflon impregnated delrin is used as the material for the compliant bushing, due to its relatively high strength and desirable deformation characteristics. Synchronous belts are Gates GT type.

preload, and significantly increased off-axial stiffness, at a cost of high assembly and disassembly difficulty. The authors do not recommend this approach unless absolutely required by application. Testing of the akr gravity compensation scheme was performed using a fixed pulley routing system, and substitution of varying stiffness springs. The fixed pulley system allows for a slight and expected variation of the output load, due to a small change in the effective distance " $a$ " as the system moves through various kinematic constraints. This variation may not be desirable, and additionally, the necessary pulley routing systems in addition to the synchronous belt to collocate the point of rotation for both akr systems significantly complicate the mechanical design. Future researchers interested in pursuing this type of mechanism are encouraged to pursue alternate methods of routing forces to the akr mechanism, such as Bowden cables (Schiele, 2008).

\section{Conclusions}

This mechanism provides a new mechanical solution that allows for a planar exoskeleton joint with the ability to carry high forces and torques, independently of the load applied to the user. It allows the transmission of very high forces through an exoskeletal joint that requires minimal effort to align. It allows the use of physical human-robot interfaces that are appropriate to the application, without need to accommodate for kinematic misalignment. The basic mechanism as described can be tailored to accommodate any desired degree of Cartesian misalignment by varying a single design parameter. Further, forces and torques can be partially decoupled, the degree of which is dependent on the relative degree of misalignment accommodated.

Such a mechanism(-s) can be integrated into an exoskeleton to allow for many users to quickly don and comfortably use the system, and allows for the introduction of exoskeletal systems to a much broader user audience.

\section{Author Contributions.}

J. F. Schorsch contributed the initial design concept, development of the technical design requirements, analysis of the mechanism, and preparation of the manuscript, with assistance from all co-authors. A. Q. L. Keemink provided analysis of the self-alignment mathematics, and many contributions to the manuscript preparation. A. H. A. Stienen assisted with the development of the concept, development of the requirements, review of prior literature, as well as technical recommendations for implementation and construction. D. A. Abbink provided many contributions to the preparation of the manuscript.

Acknowledgements. We would like to thank J. Herder, Emile Rosenberg, and Guiseppe Radaelli for their insights into the function of spring compensated mechansisms.

Edited by: D. Dodou

Reviewed by: two anonymous referees 


\section{References}

Argo: Rewalk, available at: http://www.argomedtec.coml (last access: 17 September 2012), 2012.

Berkeley Bionics: eLegs, available at: http://berkeleybionics. comlexoskeletons-rehabmobility/ (last access: 17 September 2012), 2012.

Colombo, G., Joerg, M., Schreier, R., and Dietz, V.: Treadmill training of paraplegic patients using a robotic orthosis, J. Rehabil. Res. Dev., 37, 693-700, 2000.

De Rossi, S. M. M., Vitiello, N., Lenzi, T., Ronsse, R., Koopman, B., Persichetti, A., Vecchi, F., Ijspeert, A. J., van der Kooij, H., and Carrozza, M. C.: Sensing pressure distribution on a lowerlimb exoskeleton physical human-machine interface, Sensors, 11 207-227, 2011

Evryon: Evryon, available at: www.evryon.eu/ (last access: 17 September 2012), 2009.

Jezernik, S., Colombo, G., Keller, T., Frueh, H., and Morari, M.: Robotic orthosis lokomat: a rehabilitation and research tool, Neuromodulation, 6, 108-115, 2003.

Kumar, S.: Theories of musculoskeletal injury causation, Ergonomics, 44, 17-47, 2001

Lockheed Martin: HULC, available at: http://www.lockheedmartin. com/us/products/hulc.html (last access: 17 September 2012), 2012.

Makinson, B. J.: Research and Development Prototype for Machine Augmentation of Human Strength and Endurance, Hardiman I Project, General Electric Report S-71-1056, Schenectady, NY, 1971

Morita, T., Kuribara, F., Yuki, S., and Sugano, S.: A Novel Mechanism Design for Gravity Compensation in Three Dimensional Space, Proceedings of the 2003 IEEE/ASME International Conference on Advanced Intelligent Mechatronics, 163-168, 2003.

Pratt, G. A., Williamson, M. M., Dillworth, P., Pratt, J., and Wright, A.: Stiffness Isn't Everything, Experimental Robotics IV, edited by: Salisbury J. K. and Khatib O., Springer, Berlin, 263-262, 1997.

Schiele, A.: Performance difference of Bowden Cable relocated and non-relocated master actuators in virtual environment applications, 2008 IEEE/RSJ International Conference on Intelligent Robots and Systems, IROS, 3507-3512, 2008.
Schiele, A.: Ergonomics of Exoskeletons: Subjective Performance Metrics, The 2009 IEEE/RSJ International Conference on Intelligent Robots and Systems, St. Louis, USA, 480-485, 2009.

Schiele, A. and van der Helm, F. C. T.: Kinematic design to improve ergonomics in human machine interaction, IEEE Trans. Neural Syst. Rehabil. Eng., 14 456-469, 2006.

Stienen, A. H. A., Hekman, E. E. G., Van der Helm, F. C. T., Prange, G. B., Jannink, M. J. A., Aalsma, A. M. M., and Van der Kooij, H.: Dampace: dynamic force-coordination trainer for the extremities, Proceedings of the 2007 IEEE Conference on Rehabilitation Robotics, Noordwijk, The Netherlands, 2007.

Stienen, A. H. A., Hekman, E. E. G., van der Helm, F. C. T., and van der Kooij, and H.: Self-Aligning Exoskeleton Axes Through Decoupling of Joint Rotations and Translations, IEEE Trans. Robot., 25, 628-633, 2009.

Stormont, T. J., An, K. N., Morrey, B. F., and Chao, E. Y.: Elbow joint contact study: comparison of techniques, J. Biomech., 18 , 329-336, 1985.

Tuijthof, G. J. M., and Herder, J. L.: Design, actuation and control of an anthropomorphic robot arm, Mech. Mach. Theory, 35, 945962, 2000.

Van Der Kooij, H., Veneman, J. F., and Ekkelenkamp, R.: Compliant Actuation of Exoskeletons, Mobile Robots: towards New Applications, edited by: Lazinica, A., 129-148, 2006.

Wildenbeest, J. G. W., Abbink, D. A., and Schorsch, J. F.: Haptic transparency increases the generalizability of telemanipulated motor learning, IEEE World Haptics Conference, Daejeon, Korea, 707-712, 2013.

Raytheon: XOS 2, available at: http://www.raytheon.com/ newsroom/technology/rtn08_exoskeleton/ (last access: 17 September 2012), 2012.

Zoss, A. B., Kazerooni, H., and Chu, A.: Biomechanical Design of the Berkeley Lower Limb Exoskeleton (BLEEX), IEEE/ASME Trans. Mechatronics, 11, 128-138, 2006. 